\title{
Multimodality Imaging in Clinical Nuclear Medicine
}

\author{
Jianqiao Luo* \\ Virginia Commonwealth University Healthcare Systems, USA
}

Submission: February 18, 2017; Published: February 27, 2017

*Corresponding author: Jianqiao Luo, Medical Physicist, Virginia Commonwealth University Healthcare Systems, Gateway 236, 1200 East Marshall Street, Richmond, Virginia, 23298-0001, USA, Tel: 804-828-1443; Fax: (804) 828-4181; Email: jluo@vcuhealth.org

\section{Opinion}

Positron Emission Tomography (PET) and Computed Tomography (CT) have been combined to build a hybrid or multimodality imaging system for functional imaging of physiological process and for quantification of distribution of biological tracer. The system uses high resolution CT scan to generate attenuation coefficients for attenuation correction and organ definition.

Single Photon Emission Computed Tomography (SPECT) combines functional gamma ray imaging with transmission scan of X ray CT to increase image sensitivity and contrast. The SPECT CT system has been installed in many nuclear medicine facilities and significantly changed clinical applications of the radionuclide imaging and therapy as well.

PET is based on coincidence detection of paired $511 \mathrm{keV}$ gamma rays generated from positron-electron annihilation in tissue where the positron tagged pharmaceutical distributed. Fluorine 18 (F-18) Fluoro deoxyglucose (FDG) is the most widely used radiopharmaceutical in clinical applications. Another PET imaging agent is Gallium-68 (Ga-68) for primary prostate cancer staging, small-size PSMA-positive lymph nodes and likely bone metastases. Major improvements in PET imaging equipment are new scintillation crystals; time of flight imaging and multimodality cameras: PET CT and PET MRI.

BGO, (Bismuth germinate Bi4Ge3012) used to be the crystal for dedicated PET scanners until being replaced by new generation of crystals: LSO (Cerium-doped Lutetium Orthosilicate.), GSO (Cerium-doped Gadolinium Oxyorthosilicate), and LYSO (Cerium-doped Lutetium Yttrium Orthosilicate) which are much faster scintillation materials. Although the scintillation efficiency of BOO is only about $15 \%$ of that of $\mathrm{NaI}(\mathrm{Tl})$, its significantly better detection efficiency due to high density and high atomic number has made it the choice for the detection of $511 \mathrm{keV}$ radiations, despite the fact that their relatively long decay constant of 300 ns limits coincidence timing resolution.LSO and other scintillation crystals with short decay time make it possible to implement time-of-flight detection in PET imaging, in which opposing detectors measure the difference in the arrival times of a pair of $511 \mathrm{keV}$ gamma rays. In this way, the location of the positron event can be localized within few nanoseconds along the line connecting the 2 detectors. Thus the new generation of scintillation crystals provide high stopping power for $511-\mathrm{keV}$ photons, improved scintillation light output and short decay time for possible time of flight detection. Those features result in higher count rate, lower the random coincidence rate with a tight coincidence timing window, therefore improved system spatial resolution. It also changed acquisition from 2- dimensional (2D, with septa) to 3-dimensional (3D) imaging (septa removed), which yield high noise equivalent count rate (NECR).

Supported by advanced computer technology, high speed image processing workstations provide ability of using iterative reconstruction techniques to replace analytic algorithms such as filtered back projection in clinical PET imaging. The iterative algorithm has been applied in clinical PET image reconstruction to include physics modeling of real imaging system. It has significantly improved contrast and spatial resolution especially for large size patients and small lesions as well.

To take the advantages of PET in functional imaging and $\mathrm{X}$ ray computed tomography CT in anatomical definition, combined PET and CT (PET-CT) systems have replaced PET alone in most nuclear medicine facilities. The CT portion of PET-CT provides the anatomic information through transmission scan similar to regular CT or diagnostic CT imaging. However, the CT imaging in PET-CT system normally runs under low dose mode since the transmission scan is primarily used for attenuation correction and anatomic definition but not for diagnostic purposes. These PET CT scans provide clinicians with PET, CT and fused images of both modalities for better interpretation of clinical data. Along with the success of the PET CT system, PET MRI has been introduced into clinical studies, which combines the unique 
features of PET imaging and MRI scans. Those multimodality imaging systems have shown great feature in medical imaging.

A planar image is a 2-D picture of 3-D distribution of radioactivity. Tomographic imaging is to generate a 3-D picture of the 3-D distribution. In order to collect 3-D data, a SPECT camera scans patient from multiple angles (for example: 180 or 360 degree) to generate a set of 2-D images (projections). Those 2-D projection images form the basis for reconstruction. Similar to X-ray CT. tomography, SPECT utilize image reconstruction from multiple projection images to provide a set of images (for example: transverse slices) to represent 3-D distribution. A key different between the SPECT and the X-ray CT is the SPECT is emission tomography and the X-ray CT is transmission tomography. This is due to the fact that Gamma camera and SPECT scanner detect gamma rays emitted from patient and the $\mathrm{X}$-ray pass through patient body before being detected.

In modern SPECT Camera Design,there are single head, dual-head and triple-head configurations. The dual-head SPECT cameras are the most widely used for clinical services.

The dual-head detectors can be arranged 180 degree, or 90 degree (for cardiac scan), or other angles for certain imaging protocols. Those configurations are to position the detector heads close to the patient for better special resolution.

Filtered back projection is an image reconstruction method to mathematically construct a 3-D image based on the multiple projection date from tomographic scan. Iterative reconstruction is developed to improve image quality by including physics characters in the imaging process. It has been used as major reconstruction method in emission tomography. Mathematical filters are used in the image reconstruction and processing to remove noise and select useful components in image data by filtering some spatial frequency out. It is an important part of imaging processing to select a filter and set up filter parameters in clinical nuclear medicine imaging.

SPECT imaging can provide quantitative information for data analysis. Those quantitative data can be used for dosimetry and diagnostic evaluation of disease. Therefore a SPECT image provides pictures and quantitative data to describe 3-D distribution of radionuclides in patient body. It is essential to maintain quality of the SPECT and gamma camera images to the level that current technology can provide. This involves quality product by manufacturer, equipment quality assurance and right clinical protocol by nuclear medicine service.

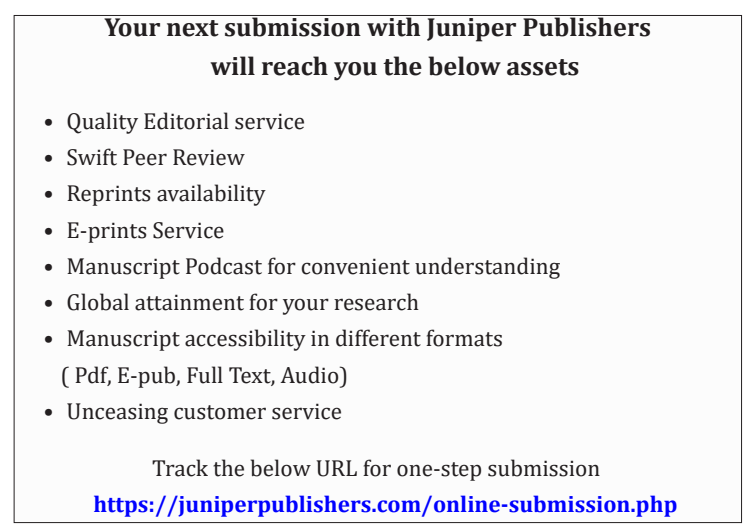

